\title{
Modeling the Local Bubble Using Multiple Supernova Remnants
}

\author{
R.K. Smith ${ }^{1}$ and D.P. Cox ${ }^{2}$
}

1 Laboratory for Astronomy and Solar Physics, NASA Goddard Space Flight Center, Greenbelt, Maryland 20771, USA

2 Department of Physics, University of Wisconsin-Madison, Madison, Wiscon$\sin 53706$, USA

\begin{abstract}
We have modeled the Local Bubble (LB) using a one-dimensional hydrocode (ODIN) that can simulate multiple supernova remnants, with non-equilibrium ion evolution and dust. Our model assumes that the local interstellar medium was a cool $\left(10^{4} \mathrm{~K}\right)$ gas approximately $5-10 \mathrm{Myr}$ ago; it was then disturbed by 2 or 3 supernovae exploding within 20-30 pc of each other over a period of 2-4 million years. The LB is the leftover hot gas from these explosions. The model predicts the $\mathrm{x}$-ray emission from such a bubble, as well as ionic abundances for hot gas ions such as O VI. These are compared to the soft x-ray data from the Wisconsin all-sky survey and the ROSAT PSPC.
\end{abstract}

\section{Introduction}

The "displacement model" is a very successful explanation of the soft x-ray background (Snowden et al., 1990); it postulates the existence of a bubble of hot $\left(10^{6} \mathrm{~K}\right)$ gas in collisional ionization equilibrium, surrounding the Sun and creating the soft x-ray background. This hot gas "displaces" the neutral gas that might otherwise be present, and so this model dovetails nicely with the observations of a local "hole" in neutral hydrogen (Paresce 1984).

While successful, understanding the physical mechanism for creating this "Local Bubble" of hot gas has proven to be difficult. Innes \& Hartquist (1984) fit the data with an analytic model of a superbubble, but they needed a very high explosion energy and external pressure. Numerical attempts have been made by Edgar (1986), who found that an active blast wave propagating in a cavity could generate the observed X-rays but over-produced OVI, and Edgar \& Cox (1993), who attempted to model the Local Bubble as a old supernova remnant (SNR). This "old SNR" model failed because when a sufficiently large bubble was created it always had a temperature too low to create the observed X-rays, unless, again, the external ISM pressure was unrealistically high. To solve this problem, we assumed the Local Bubble is the long-lived remnant of a series of supernova explosions; such a remnant can easily be made to be both large and hot, and uses the inertia of the remnant walls to confine the bubble. 


\section{Method}

We developed a one-dimensional Lagrangian hydrocode, ODIN, for this project; it is described more fully in Smith \& Cox (1997). The code divides the ISM into mass parcels and follows each mass parcel throughout time, evolving the hydrodynamic parameters as well as the ionic abundances of the gas. The ionic abundances and the non-equilibrium cooling are calculated using the Raymond \& Smith (1977) plasma spectral evolution code. Magnetic fields are included, but only as a pressure term and we assume that the field is frozen into the gas so that $|\mathbf{B}| \propto n$.

We included thermal conduction in ODIN, and found that the thermal conduction flux is one of the most important processes in fitting the observations. We used the Spitzer (1956) prescription for the thermal conduction flux:

$$
F_{t c}(T)=c_{t c} \beta T^{5 / 2} \frac{\partial T}{\partial r}
$$

where $T$ is the temperature of the gas and $\beta=6 \times 10^{-7} \mathrm{cgs} . c_{t c}$ is a unitless constant which allows for a simple "tuning" of the amount of thermal conduction in the code. We also included the effects of saturation as described by Cowie \& McKee (1977).

\section{Results}

We ran the series of models shown in Table 1, designed to explore a reasonable range of parameter space. We considered models with 2 or $3 \mathrm{SN}$ of standard $\left(\sim 10^{51} \mathrm{erg}\right)$ energy; for the $2 \mathrm{SN}$ models, the explosions were separated by one million years, and the same was true for the $3 \mathrm{SN}$ models except the third explosion occurred 2 million years after the second. We assumed the interstellar medium was homogeneous, with temperature $2 \times 10^{4} \mathrm{~K}$ and densities of order $0.2-0.4 \mathrm{~cm}^{-3}$. Our goal was to find a range of parameters that would give a long-term fit to the observations.

After the final SN explosion, each "bubble" evolves similarly, rapidly reaching maximum size and collapsing. The ISM pressure $\left(\sim 2 \times 10^{4} \mathrm{~cm}^{-3} \mathrm{~K}\right)$ exceeds the interior pressure throughout the collapse, and the rate of collapse $(\sim 10 \mathrm{~km} / \mathrm{s})$ is approximately the ISM sound speed. As Table 1 shows, the $\mathrm{X}$-ray brightness but not hardness is affected by the ISM pressure. During the collapse the center of the bubble remains at a constant temperature while the edges cool, although the bubble collapse is not entirely due to cooling; the density inside the hot bubble does increase during the collapse. As a result, the x-ray emission increases in time, as shown in Figure 1, after the impulsive peaks of the explosions.

We found that thermal conduction was necessary to smooth out the temperature distribution of the gas; this agrees with observations which find that ratios of soft $\mathrm{x}$-ray bands such as the ROSAT R1 and R2 bands are generally 
Table 1. Model parameters and general results

\begin{tabular}{|c|c|c|c|c|c|c|}
\hline Model & & $n\left(\mathrm{~cm}^{-3}\right)$ & $E_{51}\left(10^{51}\right.$ ergs $)$ & $c_{t c}$ & $\overline{\mathrm{B}(\mu \mathrm{G})}$ & Result \\
\hline $\mathrm{A}$ & 2 & 0.2 & 1.0 & $1 / 6$ & 5.0 & Reasonable fit for $\mathrm{t}=5-8 \mathrm{Myr}$ \\
\hline B & 3 & 0.4 & 1.0 & $1 / 6$ & 5.0 & Reasonable fit for $t=5-8 \mathrm{Myr}$ \\
\hline $\mathrm{C}$ & 2 & 0.2 & 0.5 & 1 & 5.0 & Too dim, too soft \\
\hline $\mathrm{D}$ & 3 & 0.2 & 0.5 & $1 / 30$ & 5.0 & Far too dim, otherwise good \\
\hline $\mathrm{E}$ & 3 & 0.4 & 1.0 & 1 & 5.0 & Too bright, too soft \\
\hline $\mathrm{F}$ & 3 & 0.2 & 1.0 & 1 & 7.0 & Too bright, too soft \\
\hline G & 3 & 0.4 & 1.0 & 1 & 7.0 & Too bright, too soft \\
\hline
\end{tabular}

smooth (Snowden et al., 1997). However, including the full amount of thermal conduction as predicted by Spitzer (1956) reduced the central temperature so much that the calculated x-ray flux was consistently too soft, the same problem that plagued Edgar \& Cox (1993) when using a single SNR. When we reduced the amount of thermal conduction, however, we were able to find acceptable fits.

We found two models, labeled A and B in Table 1, which fit many observed quantities; we will describe only $\mathrm{B}$ ( $\mathrm{A}$ is similar). Figure 1 shows the emission as a function of time in four bands (from McCammon et al., 1983): the Wisconsin all-sky survey boron (B) band $(E \sim 0.188 \mathrm{keV})$ and carbon (C) band $(E \sim 0.284 \mathrm{keV})$, the Wisconsin Beryllium $(\mathrm{Be})$ band $(E \sim 0.118 \mathrm{keV})$, and the ROSAT $3 / 4 \mathrm{keV}$ band. The brightness increases over time, and around $\sim 8 \mathrm{Myr}, 5 \mathrm{Myr}$ after the last SN explosion, the calculated fluxes are all within the measured values. One important characteristic of these models is the time-invariance of the band ratios. The calculated $\mathrm{C} / \mathrm{B}$ ratio is 2.5 , equal to the measured ratio, and it is nearly constant with time, just as the measured value is nearly constant on the sky. Switching to the ROSAT data, the flux in the ROSAT $3 / 4 \mathrm{keV}$ band is greater than that in the equilibrium displacement model, but is under the $2 \sigma$ upper limit for the Local Bubble emission as seen towards MBM 12 by Snowden et al.(1993). Finally, the predicted column density of OVI in the bubble and its edge is $\sim 1.0 \times 10^{13} \mathrm{~cm}^{-2}$, very close to the $1.6 \times 10^{13} \mathrm{~cm}^{-2}$ calculated by Shelton \& Cox (1994) to be in the Local Bubble.

Multiple supernova remnant models seem to be able to reproduce a broad range of observations of the Local Bubble. These models explain the observations as well as or better than the displacement model, and have the advantage of evolving from known astrophysical processes. The next step will be to include dust cooling and sputtering in the model, to see the effect of variable abundances on the results. 

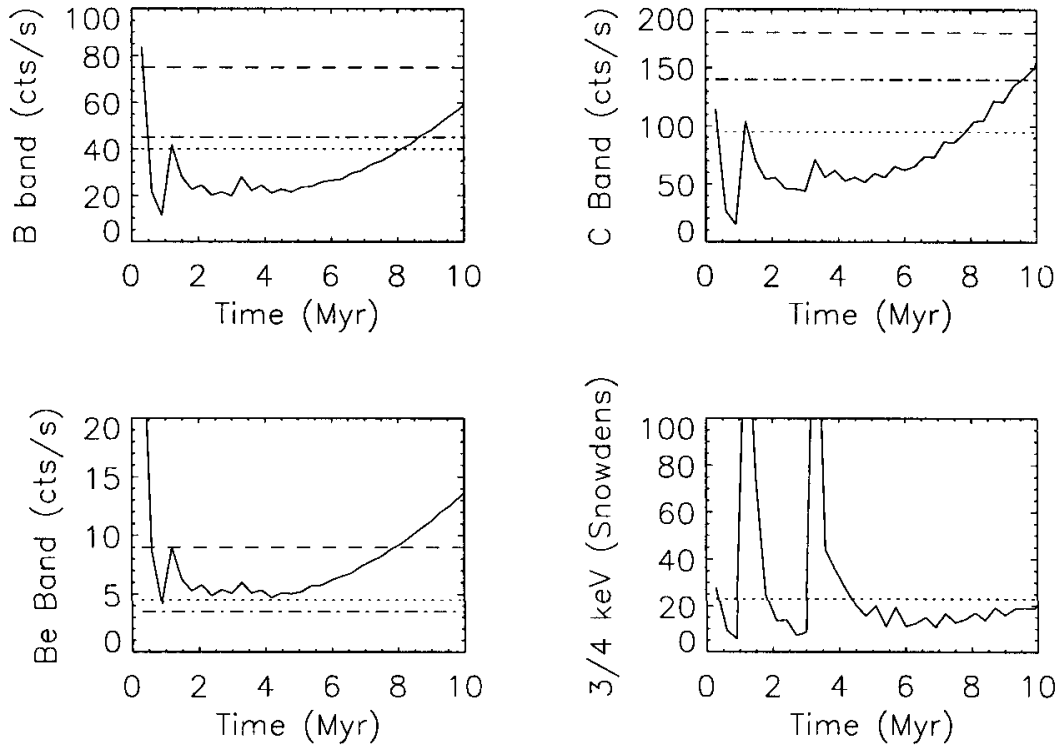

Fig. 1. The calculated emission from model B in the UW B, C, and Be bands, and the ROSAT $3 / 4 \mathrm{keV}$ band emission. For the first 3 plots, the dotted line is the observed emission in the plane. The dashed line marks the emission towards the north Galactic pole, and the dot-dashed line is the south Galactic pole emission. In the ROSAT $3 / 4 \mathrm{keV}$ plot, 1 Snowden $=10^{-6}$ counts $\mathrm{s}^{-1} \operatorname{arcmin}^{-2}$, and the dotted line marks the $2 \sigma$ upper limit from the Local Bubble as measured towards MBM 12 (Snowden et al., 1993)

\section{References}

Cowie, L. L. \& McKee, C. F. (1977): ApJ, 211, 135

Edgar, R. J. (1986): ApJ, 308, 389

Edgar, R. J. \& Cox D. P. (1993): ApJ, 413, 190

Innes, D. E. \& Hartquist, T. W. (1984): MNRAS, 209, 7

McCammon D., Burrows, D., Sanders, W. \& Kraushaar, W. (1983): ApJ, 269, 107 Paresce, F. (1984): AJ, 89, 1022

Raymond, J. C. \& Smith, B. W. (1977): ApJS, 35, 419

Shelton, R. L. \& Cox, D. P. (1994): ApJ, 434, 599

Smith, R. K. \& Cox, D. P. (1997): in preparation

Snowden S. L., Cox, D. P., McCammon, D., \& Sanders W. T. (1990): ApJ, 354, 211

Snowden S. L., McCammon, D. \& Verter, F. (1993): ApJ, 409, L21

Snowden, S. L. et al.(1997): ApJ, 485, 125

Spitzer, L. (1956): Physics of Fully Ionized Gases, (New York: Interscience) 